\title{
Cardiovascular rEmodelling in living kidNey donorS with reduced glomerular filtration rate: rationale and design of the CENS study
}

\author{
Kjersti Benedicte Blom ${ }^{a, b, c, d, e}$, Kaja Knudsen Bergo ${ }^{a, b, c, d}$, Emil Knut Stenersen Espe ${ }^{a, b, c, d}$, Vigdis Rosseland ${ }^{f}$, Ole \\ Jørgen Grøttaf, Geir Mjøeng, Anders Åsbergg, Stein Bergan ${ }^{\mathrm{h}}$, Helga Sanner ${ }^{\mathrm{i}, \mathrm{j}}$, Tone Kristin Bergersen ${ }^{\mathrm{k}, \mathrm{l}}$, Reidar \\ Bjørnerheim ${ }^{m}$, Morten Skaubyº, Ingebjørg Seljeflot',n, Bård Waldum-Grevbo ${ }^{\mathrm{e}}$, Dag Olav Dahle ${ }^{\mathrm{g}}$, \\ Ivar Sjaastad ${ }^{a, b, c, m, n}$ and Jon Arne Birkelande \\ ${ }^{a}$ Institute for Experimental Medical Research, Oslo University Hospital, Ullevål, Oslo, Norway; ${ }^{b}$ University of Oslo, Oslo, Norway; ${ }^{c} K G$ \\ Jebsen Center for Cardiac Research, University of Oslo, Oslo, Norway; ${ }^{d}$ Center for Heart Failure Research, Oslo

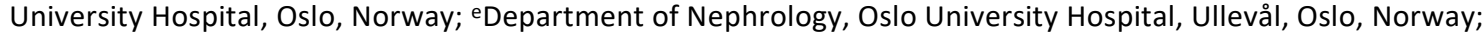 \\ fDepartment of Radiology, Division of Radiology and Nuclear Medicine, Oslo University Hospital, Ullevål, Oslo, Norway; \\ gDepartment of Transplantation Medicine, Oslo University Hospital, Rikshospitalet, Oslo, Norway; hepartment of \\ Pharmacology, Oslo University Hospital, Rikshospitalet, Oslo, Norway; 'Department of Rheumatology, Oslo University \\ Hospital, Rikshospitalet, Oslo, Norway; ${ }^{j}$ Bjørknes University College, Oslo, Norway; ${ }^{k}$ Department of Dermatology, Oslo \\ University Hospital, Rikshospitalet, Oslo, Norway; 'Institute for Clinical Medicine, University of Oslo, Oslo, Norway;

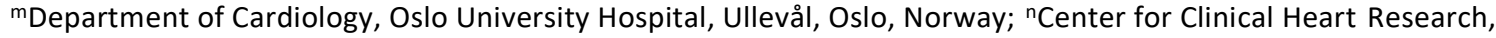 \\ Department of Cardiology, Oslo University Hospital, Ullevål, Oslo, Norway
}

ABSTRACT

Purpose: Until recently, it has been believed that donating a kidney not represents any risk for development of cardiovascular disease. However, a recent Norwegian epidemiological study sug- gests that kidney donors have an increased long-term risk of cardiovascular mortality. The pathophysiological mechanisms linking reduced kidney function to cardiovascular disease are not known. Living kidney donors are screened for cardiovascular morbidity before unilateral nephrectomy, and are left with mildly reduced glomerular filtration rate (GFR) after donation. Therefore, they represent an unique model for investigating the pathogenesis linking reduced GFR to cardiovascular disease and cardiovascular remodelling. We present the study design of Cardiovascular rEmodelling in living kidNey donorS with reduced glomerular filtration rate (CENS), which is an investigatorinitiated prospective observational study on living kidney donors. The hypothesis is that living kidney donors develop cardiovascular remodelling due to a reduction of GFR.

Materials and methods: 60 living kidney donors and 60 age and sex matched healthy controls will be recruited. The controls will be evaluated to fulfil the Norwegian transplantation protocol for living kidney donors. Investigations will be performed at baseline and after 1, 3, 6 and 10 years in both groups. The investigations include cardiac magnetic resonance imaging, echocardi- ography, bone density scan, flow mediated dilatation, laser Doppler flowmetry, nailfold capillaro- scopy, office blood pressure, 24-h ambulatory blood pressure, heart rate variability and investigation of microbiota and biomarkers for inflammation, cardiovascular risk and the cal- ciumphosphate metabolism.

Conclusions: The present study seeks to provide new insight in the pathophysiological mecha- nisms linking reduced kidney function to cardiovascular disease. In addition, we aim to enlighten predictors of adverse cardiovascular outcome in living kidney donors. The study is reg- istered at Clinical-Trials.gov (identifier: NCT03729557).

\section{KEYWORDS}

Living kidney donors; cardiovascular remodelling; cardiovascular risk; glomerular filtration rate; chronic kidney disease; vascular calcification; microcirculation

\section{Introduction and rationale}

Chronic kidney disease (CKD) is defined as persistent (>3 months) reduced kidney function (Glomerular filtration rate $(\mathrm{GFR})<60 \mathrm{~mL} / \mathrm{min} / 1.73 \mathrm{~m}^{2}$ ) and/or persistent markers of kidney damage, such as albuminuria [1]. CKD is highly prevalent and strongly associated with an increased risk of cardiovascular disease and mortality [2]. Increased cardiovascular and all-cause mortality is seen from an early stage of CKD, when GFR declines below approximately 75 $\mathrm{mL} / \mathrm{min} / 1.73 \mathrm{~m}^{2}$, and a multiplicative risk is seen with albuminuria [2]. Overall, patients with early stages of CKD are much more likely to die than to progress to kidney failure [3]. In patients on dialysis, 
the risk of cardiovascular death is ten-20 times higher that of the general population [4]. More than $40 \%$ of the mortality in end-stage renal disease is attributable to cardiovascular disease, mostly due to non-athero- sclerotic heart disease such as sudden cardiac death, arrhythmias and congestive heart failure [5]. In patients with advanced CKD, cardiovascular remodel- ling is frequent and includes calcification of vessels [6] in addition to myocardial hypertrophy and fibrosis [7]. It has been found that more than $70 \%$ of patients starting dialysis have signs of left ventricular hyper- trophy (LVH) [8]. Recent studies indicate that the remodelling process starts at early stages of CKD [9]. Edward et al. reported reduced systolic function and increased myocardial fibrosis in CKD patients with a mean GFR value of about $50 \mathrm{~mL} / \mathrm{min} / 1.73 \mathrm{~m}^{2}$ using echocardiography and T1 mapping magnetic resonance imaging (MRI), respectively [9]. This indicates that early stage CKD may contribute to the development of cardiovascular disease. The causal relationship between reduced GFR and the development of cardiovascular disease is, however, unclear as patients with CKD often have comorbidities that contribute to cardiovascular remodelling [10]. Nevertheless, in meta-analyses controlling for other cardiovascular risk factors, such as diabetes mellitus and hypertension, CKD remains inde- pendently associated with cardiovascular risk $[11,12]$.

Kidney transplantation is considered the best treatment option for patients with end-stage renal disease [13], preferably with a living donor graft as this offers superior graft quality and graft survival compared to kidneys from diseased donors [5]. After unilateral nephrectomy, living kidney donors experience an acute $50 \%$ reduction in GFR. After a few months, GFR grad- ually rises to reach $70 \%$ of pre-donation value due to hypertrophy of the remaining kidney [14]. Meticulous health screening programmes ensure that living kidney donors are healthy before donation, to mitigate pos- sible risks incurred by unilateral nephrectomy. Thus, living kidney donors represent a population with lim- ited confounding comorbidity, ideal to study the effects of early stage reduced kidney function on cardiovascu- lar remodelling and cardiovascular outcomes. Such studies are pertinent not only to clarify potential long- term risks for living kidney donors, but also to inform on prognosis for patients with CKD in general, and potentially identifying novel targets for CKD-directed cardiovascular therapy.

Previous epidemiological studies have not found increased cardiovascular or all-cause mortality in the follow-up of living kidney donors [15-20], and thus, kidney donation has been considered safe. However, most studies are retrospective and have compared kidney donors with a control group from the general population $[15,16,20]$, which may bias the results as the living kidney donors are selected to be above average healthy. A few studies have, however, used control groups fulfilling the criteria for being kidney donors [17-19,21]. Most of these studies did not find increased risk for cardiovascular disease in kidney donors [17-19], but the follow-up time was short, approximately 6 years. In 2014 Mjøen et al. could demonstrate increased risk of all-cause and cardiovas- cular mortality in kidney donors after a median fol- low up time of 15 years compared to a control group also fulfilling the criteria for being a donor [21]. Moreover, this increased mortality did not emerge until 10 years post donation. These findings again indicate that there may be a connection between reduced GFR and cardiovascular disease, but the potential mechanisms are not known. Several patho- physiological mechanisms have been proposed, and we will discuss some of these which we aim to inves- tigate in the present study.

Hypertension, which in the general population is associated with an increased risk of cardiovascular mortality [22], is one of the proposed mechanisms of increased cardiovascular risk in patients with CKD. Studies regarding development of hypertension in patients due to reduced GFR after unilateral nephrectomy have, however, reported inconsistent results. In a meta-analysis from 2006 including 48 studies with 5145 donors a possible systolic blood pressure increase of $6 \mathrm{mmHg}$ more than anticipated in the general population within 5-10 years after donation was found [23]. However, most of the studies were retrospective and about $30 \%$ of donors were lost to follow- up [23]. In addition, most of the controls were not included simultaneously with donors [23]. Also, there is a risk of surveillance bias since the donors were fol- lowed more closely than the controls. A prospective study by Kasiske et al. in 2015 showed no significant difference in visit and 24-h ambulatory blood pressure 3 years after donation, comparing 126 kidney donors with a control group of 135 individuals [24]. In con- trast, an increased risk of being diagnosed with hyper- tension in a donor group of 1278 individuals after a median follow-up of 6.2 years compared to healthy controls, has been reported [19]. Thus, hypertension might be a contributing factor to development of cardiovascular disease in patients with CKD.

LVH is a cardiovascular risk factor of great significance [25]. Studies have shown association between reduced kidney function and $\mathrm{LVH}$; both independent 


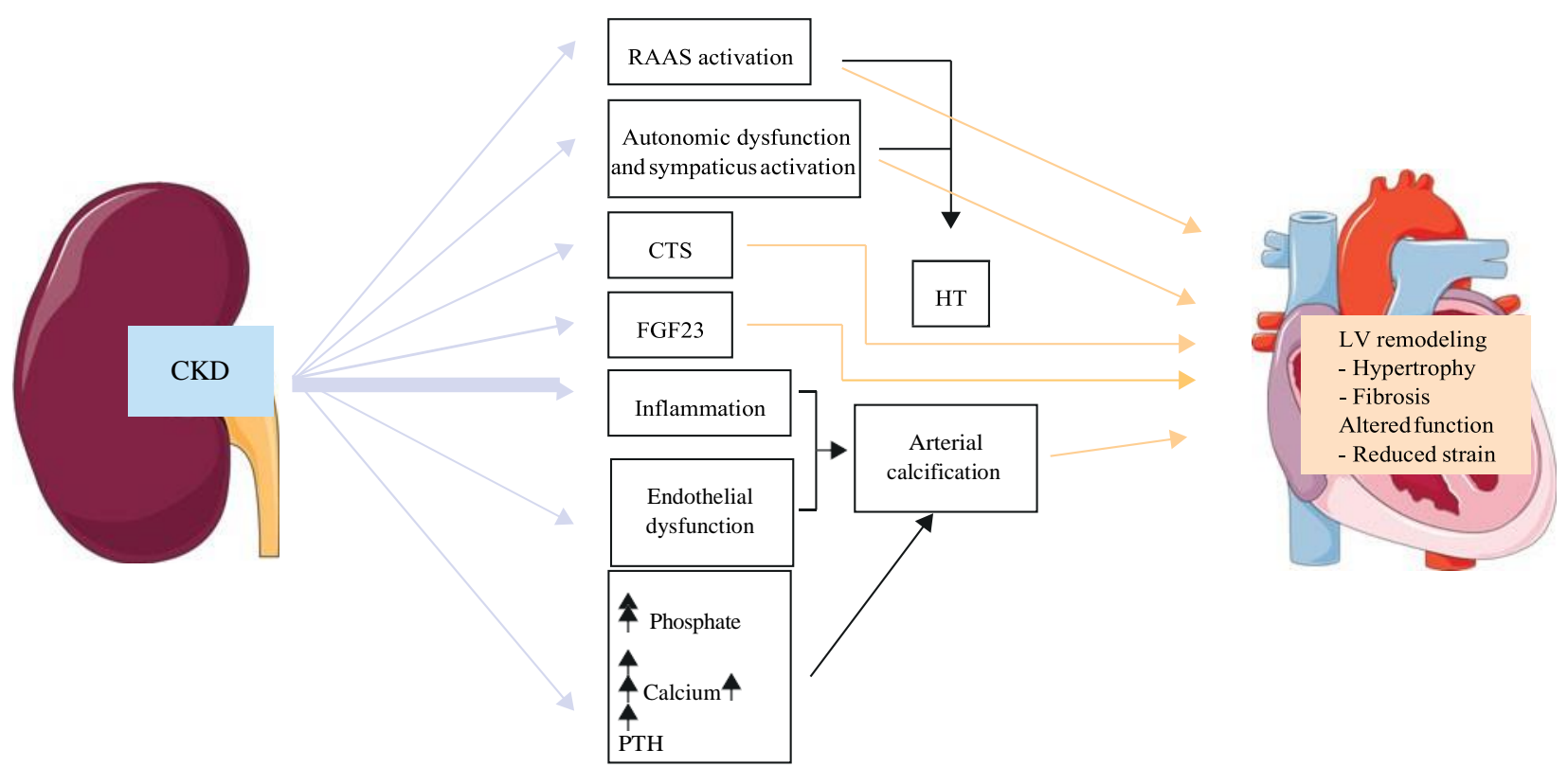

Figure 1. Potential pathophysiological mechanisms of cardiovascular remodelling in CKD patients. Abbreviations: CKD: chronic kid-ney disease; RAAS: renin-angiotensin-aldosterone-system; CTS: cardiotonic steroids; FGF23: fibroblast growth factor 23; PTH: para- thyroid hormone; HT: hypertension; LV: left ventricle. The figure contains elements modified from Servier medical by Servier, which is licensed under a Creative Commons Attribution 3.0. Unported License.

of [26], and associated, with hypertension [27]. Also, a study of 31 kidney recipients found reversal of LVH after improvement of renal function post transplantation [28]. A few studies have reported altered cardiac structure and/or function after unilateral nephrectomy [29-31]. Both Altmann et al. and Moody et al. found increased left ventricular mass corresponding to the reduction in GFR without an increase in blood pressure 1 year after unilateral nephrectomy [29,30], suggesting that the remodelling process takes place through other pathophysiological mechanisms than hypertension.

In addition to cardiac remodelling, CKD is associated

with accelerated medial and intimal calcification [6], representing accelerated arteriosclerosis and atheroscler-osis, respectively. Hyperphosphatemia, hypercalcemia and increased levels of parathyroid hormone might con- tribute to medial calcification by causing a shift from vascularsmoothmusclecells to osteoblast-like cells [32]. Vascular calcification leads to arterial stiffening, which again contributes to development of fibrosis andLVH. Inflammation and endothelial dysfunction are also sug- gested to be important factors contributing to develop- ment of vascular calcification. Both inflammation [33] and endothelial dysfunction [34] are associated with end-stage renal disease, and proposed to contribute to increased cardiovascular mortality $[35,36]$.

Neurohormonal mechanisms such as inappropriate activation of the renin-angiotensin-aldosteronesystem are proposed as potential contributing

Factors in the development of cardiovascular disease in CKD.
Medication that inhibits the renin-angiotensinaldoster- one-system have shown to reduce development of left ventricular remodelling in patients with essential hyper-tension [37] and the risk of cardiovascular events in patients with renal insufficiency [38]. In patients with CKD, angiotensin converting enzyme inhibitors reduce the risk for endstage renal disease, cardiovascular events and allcause mortality [39]. Circulating levels of renin and aldosterone have not been found elevated after kidney donation [29,31]. However, in addition to the circula- tory effects of the renin-angiotensinaldosterone-system activations, studies have also suggested the importance of intracellular reninangiotensin-aldosterone-system effects [40]. Urinary angiotensinogen has been proposed as a novel biomarker of the intrarenal renin-angiotensinaldosterone-system and a study of 20 living kidney donors found increased levels of urinary angiotensinogen 12 months after unilateral nephrectomy [41]. Also, the autonomic nervous system and the kidneys are known to interplay [42]. Autonomic dysfunction has shown to associate with cardiovascular remodelling [43] and increased risk of early death in patients with end-stage renal disease [42].

Fibroblast growth factor 23 (FGF23) is another potential factor that might contribute to development of LVH in CKD [44]. FGF23 is synthesised in response to increased levels of phosphate and binds to the FGF receptor and its associated co-receptor 


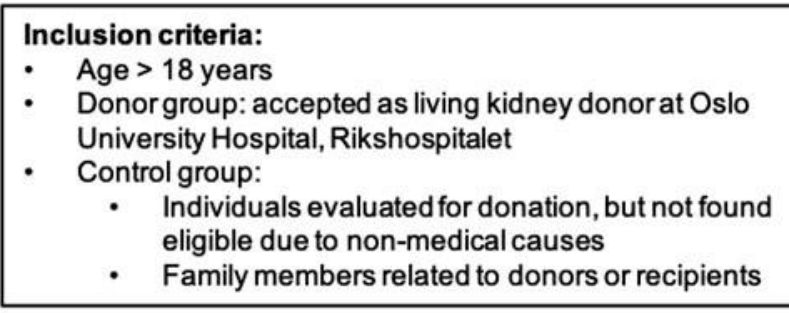

Figure 2. Inclusion and exclusion criteria. Abbreviations: CVD: cardiovascular disease; BP: blood pressure; BMI: body mass index; mGFR: measured glomerular filtration rate; ECG: electrocardiogram; HIV: human immunodeficiency virus; TB: tuberculosis; HBV: hepatitis B; HCV: hepatitis C; EBV: Epstein-Barr virus.

Klotho in renal tubular cells [44]. Activation of the FGF receptor complex causes excretion of phosphate in urine by inhibiting reabsorption of phosphate [44]. CKD is associated with elevated circulating levels of FGF23, which increase in early stages of CKD, even before phosphate and parathyroid hormone levels increase [44]. Studies have reported on an association between FGF23 and LVH in humans [44], including kidney donors [29]. In patients starting haemodialysis, FGF23 levels were found to be independently associ- ated with mortality [45]. The FGF receptor cofactor, Klotho, is produced mainly by the kidneys. Circulating klotho, which protects the heart against stress induced maladaptive hypertrophy and remodel- ling, has been shown decreased in patients with CKD [44]. Thus, both FGF23 and klotho might play a role in cardiac remodelling during CKD.

Another interesting group of signalling molecules are the cardiotonic steroids. Cardiotonic steroids plasma levels are increased in patients with CKD and elevated cardiotonic steroids plasma levels are associ- ated cardiovascular remodelling [46]. Cardiotonic ste- roids inhibit the $\mathrm{Na}^{\mathrm{b}} / \mathrm{K}^{\mathrm{b}}$ ATPase and thus might increase intracellular cardiomyocyte $\mathrm{Na}^{b}$ and $\mathrm{Ca}^{2 \mathrm{~b}}$ concentrations [46]. High intracellular cardiomyocyte

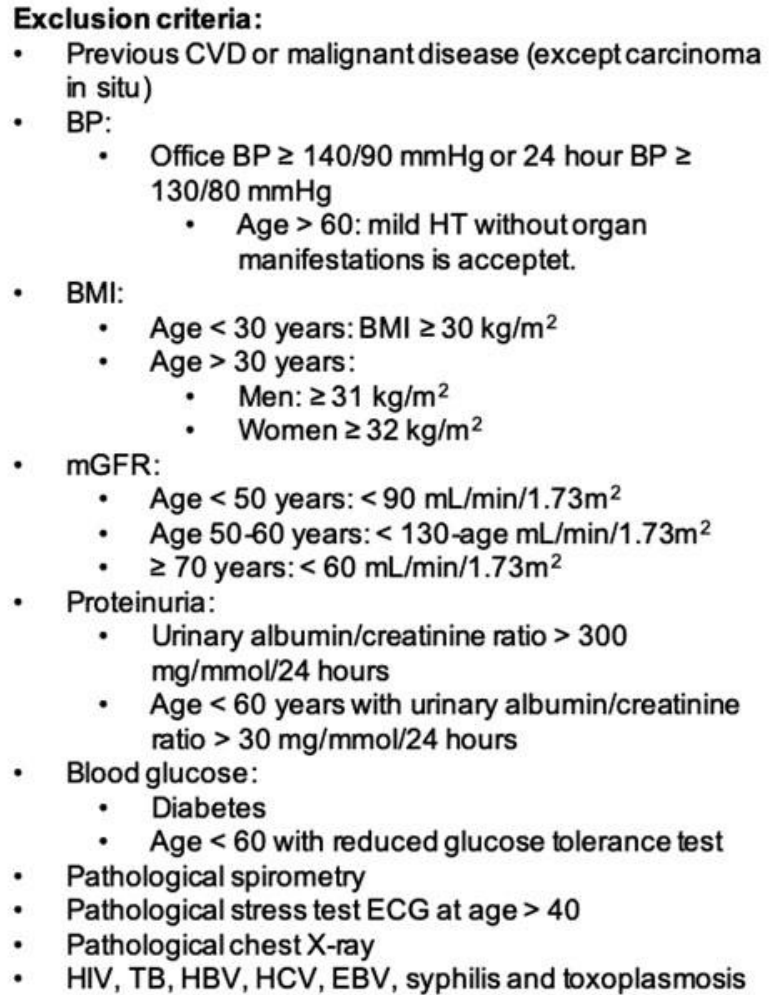

-

$\mathrm{Ca}^{2 \mathrm{~b}}$ concentrations are associated with increased risk of arrhythmias and sudden cardiac death in patients with heart failure [47] (Figure 1).

The aim of the CENS study is to investigate the above-mentioned potential pathophysiological mech- anisms of cardiovascular remodelling potentially induced by a reduction of GFR, utilising living kid- ney donors who experience an acute drop in kidney function, compared to age matched subjects with normal kidney function. We also aim to identify pre- dictors of adverse cardiovascular remodelling in these patients. This can help us to better select appropriate kidney donors, to give the donors cor- rect information about the consequences of kidney donation, and perhaps pinpoint important risk reducing factors.

\section{Methods and design}

\section{Study population and design}

The project is a prospective longitudinal parallel group study including subjects accepted as living kidney donors according to the Norwegian donor criteria at Oslo University Hospital - Rikshospitalet, Norway 


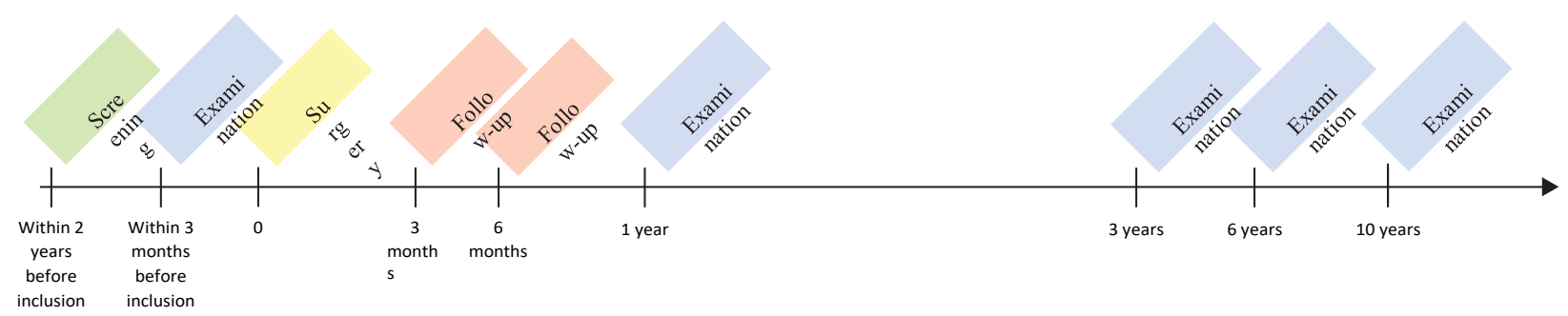

Screening
- Medical history and clinical examination
- Office BP
- mGFR
- Blood tests
- Urine microscopy and biochemistry
- Glucose tolerance test
- Chest x-ray
- ECG
- Stress test ECG at age > 40 years or CV risk

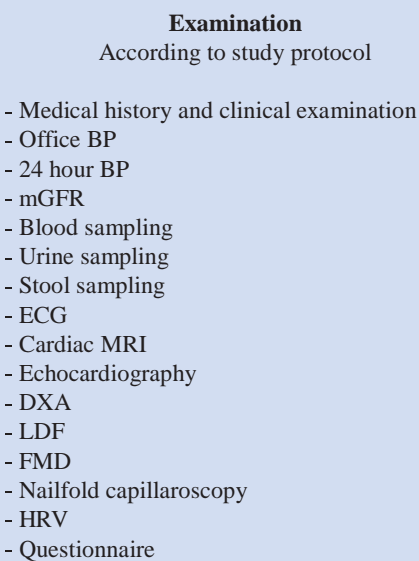

Examination

According to study protocol

- Medical history and clinical examination

- Office BP

-24 hour BP

- mGFR

- Blood sampling

- Urine sampling

- Stool sampling

- ECG

- Cardiac MRI

- Echocardiography

- DXA

- LDF

- FMD

- Nailfold capillaroscopy

- HRV

- Questionnaire

Follow-up of living kidney donors At local nephrologist

- Medical history and clinical examination - Office BP

- Blood tests

Urine biochemistry

Figure 3. CENS study timeline. Abbreviations: BP: blood pressure; mGFR: measured glomerular filtration rate; ECG: electrocardio- gram; CV: cardiovascular; MRI: magnetic resonance imaging; DXA: dual-energy X-ray absorptiometry; LDF: laser Doppler flowmetry; FMD: flow mediated dilatation; HRV: heart rate variability.

(OUH), Norway's national transplantation centre. Collaboration with local nephrologist at all regional and university hospitals in Norway has been established and the project therefore has the opportunity to invite all Norwegian living kidney donors in the inclusion period to participate in the study. OUH annually performs 70-90 transplantations using living kidney donors. The 2year inclusion period is antici- pated to result in 60 participating donors. In order to correct for naturally occurring age related changes in the cardiovascular system, the donors will be compared to an age- and sex-matched control group. Individuals evaluated for donation, but not found eli- gible due to causes not related to GFR and cardiovas- cular risk, such as immunological mismatch, will be invited to participate in the control group. The con- trol group will also include healthy family members related to donors, recipients and/or patients with CKD. Candidate family members will also be eval- uated according to the Norwegian transplantation protocol for living kidney donors, and must fulfil these criteria before inclusion as controls. Control individuals that accept to participate will go through the same test battery as living kidney donors. All participants will give written consent before inclusion (Figure 2).
Investigations according to the project protocol will be performed within 3 months before unilateral nephrectomy. Donors and controls will be reassessed after 1, 3, 6 and 10 years with the same test battery as at enrolment. In addition, the donors are followed up at 3 and 6 months after surgery by a nephrologist at their regional hospital, according to the transplant- ation protocol (Figure 3 ).

\section{Power analysis}

Our study population and study design have similar- ities to the study by Edwards et al [9], using cardiac MRI parameters in a group of CKD patients and con-trols. Based on data from the Edwards study, a power of $80 \%$ and $a<0.05$, we need to include 50-60 indi- viduals in each group. Thus, inclusion over a period of 2 years should provide a sufficient number of par- ticipants. The inclusion of control persons might be challenging and represent a risk to the project. However, risk mitigation is achieved by combining groups mentioned above, and if necessary, use the Norwegian population registry and draw individuals for screening according to the transplantation proto- col. If the criteria are fulfilled, the individuals can be included in the control group. 


\section{Study protocol}

\section{Office blood pressure}

Office blood pressure will be measured using validated automatic devices. The cuff size will be adjusted according to the arm circumference. Measurements will be performed after 5 min of rest and with the participant having refrained from tobacco, exercise and caffeine intake for the past 30 min. Measurements will be performed with the participant in a relaxed, sitting position, without crossing legs and with the cuff at heart level in a quiet room with an observer present. First, blood pressure will be measured in both arms. The arm with the highest blood pressure will be used for all subsequent measurements. Blood pressure will be measured three times with intervals of at least 1 min. Additional measurements (max-imum two) will be recorded if the last two readings differ by $>10$ $\mathrm{mmHg}$ in either systolic, or diastolic, blood pressure. The blood pressure value is deter- mined as the average of the two last measurements.

\section{Hour blood pressure}

Measurements will be done on the non-dominant arm. The cuff size will be adjusted according to the arm circumference. Blood pressure will be recorded automatically every 20 min during the day (between 07 and 22) and every 60 min at night (between 22 and 07 ). A minimum of $70 \%$ usable blood pressure readings is required. The participants will be informed to 1) fill out a diary with information about special events that occur and sleep quality, 2) keep the arm at rest and avoid movement at every blood pressure measurement, 3) do common everyday activ- ities, but avoid heavy physical activity.

\section{Cardiac magnetic resonance imaging}

Detailed assessment of myocardial structure and func- tion will be performed with a $1.5 \mathrm{~T}$ scanner (Philips Ingenia; Philips Healthcare, Best, The Netherlands) utilising several methods. A stack of short axis CINE loops as well as long axis, four chamber and left ven-tricular outflow tract CINE loop will facilitate robust measurements of global function and structural parameters, such as left ventricular and right ventricu- lar mass, stroke volumes and ejection fractions. Native T1-mapping will be used to evaluate myocar- dial fibrosis patterns. Tissue phase mapping will be used to measure the detailed $3 \mathrm{D}$ deformation of the heart, allowing comprehensive analysis of regional myocardial strain and strain rate with high resolution.
Thus, tissue phase mapping offers information about both systolic and diastolic function. Moreover, in combination with blood pressure, tissue phase map- ping allows measurement of regional myocardial work [48]. Also, the combination of T1-mapping and tissue phase mapping enables high sensitive assessment of myocardial structure-function relationships.

Pulse wave velocity is a well-established marker for aortic stiffness and is a strong and independent pre- dictor of cardiovascular mortality in patients with end-stage renal disease [49]. Assessment of aortic pulse wave velocity using flow-encoded MRI will be used to measure aortic stiffness [50].

\section{Echocardiography}

Standard and 3 D-echocardiography will be performed using a Vivid E9 cardiac ultrasound scanner (GE Vingmed Ultrasound, Horten, Norway). Myocardial function and structure will be followed over time by B-mode, M-mode, tissue doppler, blood flow doppler and strain imaging, as previously described [51].

\section{Carotid ultrasound}

Non-occlusive atherosclerotic plaques and increased carotid intima media thickness represent early vascular remodelling and is associated with increased cardiovascular risk [52]. Carotid intima media thickness will be measured after recommendations by Touboul et al. [53]. Carotid ultrasound in B-mode will be used, with a (6-15.0 MHz) linear array transducer (ML6-15-D) at frequencies $>7 \mathrm{MHz}$. The depth of focus will be about 30-40 $\mathrm{mm}$ and the optimal frame rate about $25 \mathrm{~Hz}(>15 \mathrm{~Hz})$. The common carotid artery, the carotid bulb and the internal carotid artery will be visualised on both sides in longitudinal and cross-sectional views. The carotid intima media thickness measurement will be performed with a lateral probe position on both sides in a longitudinal view, perpendicular to the ultrasound beam with both walls clearly visualised in a region free of plaques on the far wall of the common carotid artery, at least 5 $\mathrm{mm}$ below its end. An automated system will be used to provide accurate measurements of carotid intima media thickness and common carotid artery interadventitial and intraluminal diameters.

\section{Heart rate variability}

The function of the autonomic nervous system regu- lating the sinoatrial node can be assessed with heart rate variability [54]. Low heart rate variability is asso- ciated with increased mortality in patients after acute 
myocardial infarction [55]. Heart rate variability will be measured using a Polar V800 monitor [56]. RR interval data will be recorded continuously during night-time at a sampling frequency of $1000 \mathrm{~Hz}$.

\section{Nailfold capillaroscopy}

Microvascular architecture can be assessed easily and non-invasively using nailfold capillaroscopy. Changes in nailfold capillary architecture might represent changes in the microvascular architecture in other organs, including the heart. Therefore, we will per- form nailfold capillaroscopy to evaluate changes in the microvascular architecture due to a reduced GFR. Nailfold capillaroscopy will be performed after recom- mendations by Etehad Tavakol et al. [57]. The partici- pants will be asked to avoid smoking, caffeine intake and vigorous physical activity the last $6 \mathrm{~h}$ before examination. They will be seated in a quiet room with temperature of 20-24 ${ }^{\circ} \mathrm{C}$. Capillary density meas- urements will be performed with nailfold microscopy using a $200 \times$ magnification capillaroscope (Dino-Lite Pro 200x). Finger number 3,4 and 5 on both hands will be examined and three pictures will be taken of each finger. In all of the 18 pictures, the number of capillaries per millimetre will be measured, using the DinoCapture 2.0 software. The capillary density is determined as the mean of the 18 measured densities.

\section{Laser Doppler flux and flow mediated dilatation}

Laser Doppler flux enables non-invasive monitoring of the microvascular blood perfusion of the skin. The vascular endothelium possesses a potent vasodilatory mechanism lowering vascular resistance, which is believed to represent a general function of the micro- circulation, including vessels of the heart [58]. Flow mediated dilatation enables noninvasive investigation of endothelial function [59]. We will utilise laser Doppler flux and flow mediated dilatation simultan- eously to investigate endothelial function in small and medium-sized vessels, respectively. Flow mediated dilatation will be performed according to current guidelines [60].

The participants will be investigated in a quiet, darkened, room, resting in a supine position. The participants must have avoided vigorous physical activity, and be fasting (including smoking, alcohol and caf-feine intake) since the previous evening.

The laser Doppler probes (moorVMS, Moor instru- ments, Devon, UK) will be fastened with adhesive tape to the pulps of the third fingers of both hands (dominated by the shunt vessels; arteriovenous anastomoses), and $2 \mathrm{~cm}$ proximal for the wrist of the dorsal site of both hands (dominated by nutritive vessels). An automated forearm cuff will be placed below the elbow of the right arm (moorVMS-PRES, Moor instruments, Devon, UK). After fastening of the laser Doppler probes and application of the forearm cuff, the right brachial artery will be visualised in longitudinal section about $5 \mathrm{~cm}$ proximal to the antecubital fossa with a $12 \mathrm{MHz}$ linear array ultrasound probe (Zonare z.one; ZONARE Medical Systems, Mountain View, CA, USA). Simultaneously, an electrocardiogram (ECG) will be recording and instantaneous arterial blood pressure will be obtained from the left fourth finger (Ohmeda 2300 Finapres, Madison, WI). Skin temperature and room temperature will also be recorded.

After ten min of control measurements, the forearm cuff will be inflated to $200 \mathrm{mmHg}$ (above systolic blood pressure) for five min. After this, shear stress will be made by deflating the cuff and measurements is then continued for ten min after cuff deflation.

Recording will be done simultaneously from all four Laser Doppler probes with sampling frequency of $40 \mathrm{~Hz}$. The noise limitation filter of the Laser Doppler instruments will be set to the highest level $(21 \mathrm{kHz})$, and the radiated wavelength will be $820 \mathrm{~nm}$. Flux output signals will be filtered at time constant $0.1 \mathrm{~s}$. Baseline diameter of the brachial artery will be stored before occlusion. After deflation, brachial artery diameter will be stored for $180 \mathrm{~s}$.

\section{Dual-energy X-Ray absorptiometry (DXA)}

We will evaluate changes in bone mineral density and soft tissue composition using a Lunar Prodigy (Madison, WI, USA) DXA scanner to perform DXA scans of the total body, lumbar spine and hips [61].

\section{Specific bloodsamples}

Renal function will be assessed using the renal clear- ance of iohexol following a $5 \mathrm{~mL}$ iv injection of Omnipaque $^{\mathrm{v}}$ (iohexol) $300 \mathrm{mg} \mathrm{l} / \mathrm{mL}$. Routine bio- chemical parameters will be recorded and analysed by conventional methods. In addition, blood samples will be processed after standardised procedure, and serum and plasma samples aliquoted and stored at $-80^{\circ} \mathrm{C}$ in a biobank. That also includes PaxGene tubes for gene expression analyses. A number of biomarkers, including markers of inflammation, connective tissue remodelling, bone metabolism and calcification, are planned analysed. 
Faecal samples for biobank

Faecal samples will be collected using a sampling device and dry sterile tubes with spoons for fresh faeces. The samples will be aliquoted and stored dry at $-80{ }^{\circ} \mathrm{C}$ within $24 \mathrm{~h}$ after sampling.

\section{Urine samples for biobank}

Morning urine samples will be collected and centrifu-gated at $4{ }^{\circ} \mathrm{C}$ at $2500 \times \mathrm{g}$ for ten min and the super- natant will be aliquoted and kept at $-80{ }^{\circ} \mathrm{C}$ in a biobank.

\section{Ethics}

The protocol has been approved by the Regional Committee for Medical Research Ethics in December 2016 (ref. number: 2016/1961) and the study has been registered at Clinical-Trials.gov (identifier: NCT03729557). The participants will be included in the study after informed and written consent.

\section{Funding and study management}

This work is supported by the [South-Eastern Norway Regional Health Authority] under Grant [2018017]; the [Bergesen foundation]; the [Sissener foundation]; the [Fondsstiftelsen] at OUH; the [Foundation for the Promotion of Research in Kidney Diseases].

The study has a steering committee for monitoring the study progress and quality. The Steering committee consisting of Consultant Jon Arne Birkeland, Professor Ivar Sjaastad, Consultant Dag Olav Dahle, PhD-candidate Kjersti Blom and a user representative from the National Association for Kidney Disease and Transplant, Olav Bjørnsund Hollingsaeter. In addition, we will use standardised forms for patient reported outcome measures (PROMS) that will measure the health status of the participants in the study seen from a user site.

\section{Discussion}

There is strong epidemiological evidence for an association between reduced kidney function and cardiovascular disease $[2,5]$. Studies also suggest that cardiac remodelling in CKD begins early [9]. However, it is difficult to investigate the causative relationship between CKD and cardiovascular disease because of multiple confounders in this group of patients. Kidney donors represent a unique model for investigating the potential pathophysiological mechanisms because they are screened for cardiovascular morbid- ity before donation and end up with $70 \%$ of their ori- ginal GFR after unilateral nephrectomy [14]. The CENS study will examine consequences of unilateral nephrectomy and the mechanisms connecting reduced kidney function to cardiovascular disease. The main outcome of the CENS study is structural and func- tional remodelling of the heart. The secondary out- come is structural and functional remodelling of the small-, medium sized and large vessels. A sub goal of the CENS study is to investigate factors that might change due to a reduction of GFR, and potentially induce changes in the cardiovascular system.

\section{Structural and functional remodelling of the heart}

LVH is common in patients with advanced kidney disease and is associated with cardiovascular mortality [8]. The presence of cardiovascular structural and functional remodelling after kidney donation has until recently been poorly explored. This is partly due to methodological aspects, as earlier studies on cardiac remodelling in CKD have been performed using con- ventional echocardiography techniques. These meth- ods do not have the proper sensitivity to detect early structural and functional alterations in the cardiovascular system [62]. Recent studies investigating cardiac structure and function in living kidney donors with more sensitive MRI methods suggest development of small changes in the heart after unilateral nephrec- tomy [29-31]. Moody et al. even found global left ventricular remodelling as early as 1 year after kidney donation [29]. However, the relationship between altered structure and changes in myocardial function on the regional level in kidney donors is not known. The CENS study will provide novel information about cardiac remodelling by using even more sensitive MRI techniques such as $3 \mathrm{D}$ tissue phase mapping.

Native T1 mapping provides information about the microstructure of the myocardium [63], including the degree of fibrosis [64]. Several pathological processes influence T1 values, such as diffuse myocardial fibro- sis [64], oedema [65], and infiltrative diseases [66]. We hypothesise that native T1 will be altered in kid- ney donors due to development of diffuse fibrosis sec- ondary to reduced kidney function.

Native T1 mapping is often used in combination with gadolinium-based contrast agents to provide information about the extracellular volume [67] and diffuse fibrosis [68]. However, known risk factors when using gadolinium-based contrast agents include allergic reactions and nephrogenic systemic fibrosis in 
patients with advanced renal disease [69]. Also, sev- eral studies have shown signs of gadolinium depos-ition in the brain after repeated exposure to gadolinium-based contrast agents, of which the conse- quences are not known [70]. Kidney donors are healthy before kidney donation, and we do not expect changes in T1 value due to other pathology such as oedema, amyloidosis or iron overload. We can there- fore assume that, if found, an increase in native $\mathrm{T} 1$ values in living kidney donors compared to controls are due to development of interstitial cardiac fibrosis secondary to a reduction in GFR. Moreover, the added value of post-contrast T1 compared to purely native T1 is still a matter of debate [71]. Therefore, we have chosen not to expose the study participants to gadolinium-based contrast agents.

While T1-mapping provides information about myocardial structure, tissue phase mapping offers detailed information about left ventricular function. Tissue phase mapping provides strain and strain rate with very high temporal and spatial resolution [72]. This is essential for detecting subtle changes, i.e., it offers higher sensitivity. Moody et al. [29] used feature tracking, which is an MR post-processing tool used to extract deformation parameters from conven- tional CINE MR scans. However, feature tracking is less sensitive than tissue phase mapping in detecting the complex deformation of the left ventricular [73] since tissue phase mapping provides information about the direction and velocity of each point of the left ventricular myocardium for all time points. By measuring blood pressure in connection with the MRI examination, one can also calculate myocardial work by combining this with data from the tissue phase mapping investigation [48]. To our knowledge, no other study has previously investigated living kid- ney donors and a comparable control group prospect- ively using T1-mapping and tissue phase mapping. The CENS study will therefore contribute to novel information about cardiac remodelling induced by reduced GFR.

\section{Structural and functional remodelling of the large-, medium sized and small vessels}

The CENS study will also investigate arterial remodelling in large-, medium sized and small vessels. Thus, if present, adverse arterial remodelling will not only be identified, but also localised to different segments of the arterial system. This is important not only for peripheral remodelling, but also because it is a determinant of cardiac function.
Signalling mechanisms inducing cardiovascular remodelling due to $C K D$

Thus, we intend to investigate arterial and cardiovascular remodelling in living kidney donors, and further investigate the association with altered calciumphos- phate metabolism, endothelial function, inflammation parameters and autonomic nervous system function. The aim of the CENS study is to enlighten potential pathophysiological mechanisms and predictors of car- diovascular disease in patients with CKD. As far as we are aware of, no previous study has performed such novel and extensive investigation of kidney donors and an equally healthy control group. The CENS study will contribute with novel insights regarding cardiovascular remodelling due to reduced GFR in living kidney donors.

\section{Importance and clinical significance}

This knowledge is especially important for two groups. Firstly, it might contribute to more robust risk stratification, and possibly, identify risk-reducing interventions in living kidney donors. Living kidney donors make a significant allowance by contributing to the transplantation programme. Hence, they deserve the best possible medical care, and the most updated information available regarding possible adverse effects. Second, CKD patients are at increased risk of death, cardiovascular events and hospitalisa- tion compared to the general population [74]. Whether this observed increase in risk is explained by CKD or comorbidity secondary to CKD, e.g., hyper-tension, is not properly investigated, though recent studies have suggested that reduced kidney function is an independent risk factor for development of cardio- vascular disease [29]. This project will give more information about the mechanisms underlying increased risk of cardiovascular disease in patients with reduced GFR. Such knowledge might contribute to identify riskreducing interventions. Taken into account the large numbers of CKD patients among the general population, our findings, if they turn into interventions, might have a huge impact on public health.

\section{Acknowledgement}

We are grateful to medical technologists Jeanette Konstanse Steen and Sissel Åkra and nurse Charlotte Holst Hansen at Centre for Clinical Heart Research, Department of Cardiology, OUH, Ullevål, for help with participant visits and handling of patient samples. Also, we are grateful to the bioengineer Lena Bøhnsdalen Nygaard, nurses Helga 
Grimstad Sørhøy, Sebastian Muller, Kjersti Lønning, Fanny Bruserud, Janne Marlene Gripheim, Rita Gald, Kathe Meyer and Sissel Paulsen, and secretary Birgitte Klevengen, Asima Bibi and Torill Johnsen at the Department of Transplantation Medicine, OUH, Rikshospitalet, for help with participant visits. We also wish to thank Consultant Fredrik Borchsenius for facilitating access to spirometry investigation of control participants at the Department of Pulmonary medicine, OUH, Ullevål. Also thanks to section manager of the MR section, Wenche Synn $\varnothing$ ve Andreassen, and secretary Grethe Hansen at the Department of Radiology, OUH, Ullevål for help with facilitating MR investigation of participants. Finally, we wish to thank Consultant Lars Nordsletten and nurses Janne Bleken $\mathrm{H} \varnothing$ glund, Karoline Hjetland, Hilde Losnedal and Randi Kvaern $\varnothing$ at the Department of Orthopaedics, OUH, Ullevål, for help with DXA-scans of participants.

\section{Disclosure statement}

No potential conflict of interest was reported by the authors.

\section{References}

[1] Group KDIGOKCW. Clinical practice guideline for the evaluation and management of chronic kidney diseas. Kidney Int. 2012;1-150.

[2] Matsushita K, van der Velde M, Astor BC, et al. Association of estimated glomerular filtration rate and albuminuria with all-cause and cardiovascular mortality in general population cohorts: a collabora- tive meta-analysis. Lancet. 2010;375(9731): 2073-2081.

[3] Keith DS, Nichols GA, Gullion CM, et al. Longitudinal follow-up and outcomes among a population with chronic kidney disease in a large managed care organization. Arch Intern Med.. 2004; 164(6):659-663.

[4] Foley RN, Parfrey PS, Sarnak MJ. Clinical epidemiology of cardiovascular disease in chronic renal disease. Am J Kidney Dis. 1998;32(5):S112-S119.

[5] System USRD. USRDS annual data report: epidemiology of kidney disease in the United States. Bethesda (MD): National Institutes of Health, National Institute of Diabetes and Digestive and Kidney Diseases; 2018.

[6] Goodman WG, Goldin J, Kuizon BD, et al. Coronary-artery calcification in young adults with end-stage renal disease who are undergoing dialysis. N Engl J Med. 2000;342(20):1478-1483.

[7] Mall G, Huther W, Schneider J, et al. Diffuse intermyocardiocytic fibrosis in uraemic patients. Nephrology, dialysis, transplantation: official publication of the European Dialysis and Transplant Association. Eur Renal Assoc. 1990;5(1):39-44.

[8] Foley RN, Parfrey PS, Harnett JD, et al. Clinical and echocardiographic disease in patients starting endstage renal disease therapy. Kidney Int. 1995;47(1): 186-192.
[9] Edwards NC, Moody WE, Yuan M, et al. Diffuse interstitial fibrosis and myocardial dysfunction in early chronic kidney disease. Am J Cardiol. 2015; 115(9):1311-1317.

[10] Jha V, Garcia-Garcia G, Iseki K, et al. Chronic kid- ney disease: global dimension and perspectives. Lancet. 2013;382(9888):260-272.

[11] Fox CS, Matsushita K, Woodward M, et al. Associations of kidney disease measures with mortality and end-stage renal disease in individuals with and without diabetes: a meta-analysis. Lancet. 2012; 380(9854):1662-1673.

[12] Mahmoodi BK, Matsushita K, Woodward M, et al. Associations of kidney disease measures with mortality and end-stage renal disease in individuals with and without hypertension: a meta-analysis. Lancet. 2012;380(9854):1649-1661.

[13] Wolfe RA, Ashby VB, Milford EL, et al. Comparison of mortality in all patients on dialysis, patients on dialysis awaiting transplantation, and recipients of a first cadaveric transplant. N Engl J Med. 1999; 341(23):1725-1730.

[14] Krohn AG, Ogden DA, Holmes JH. Renal function in 29 healthy adults before and after nephrectomy. JAMA. 1966;196(4):322-324.

[15] Fehrman-Ekholm I, Elinder CG, Stenbeck M, et al. Kidney donors live longer. Transplantation. 1997; 64(7):976-978.

[16] Ibrahim HN, Foley R, Tan L, et al. Long-term consequences of kidney donation. N Engl J Med.. 2009; 360(5):459-469.

[17] Garg AX, Meirambayeva A, Huang A, et al. Cardiovascular disease in kidney donors: matched cohort study. BMJ. 2012;344(mar01 2):e1203.

[18] Segev DL, Muzaale AD, Caffo BS, et al. Perioperative mortality and long-term survival fol- lowing live kidney donation. JAMA. 2010;303(10): 959-966.

[19] Garg AX, Prasad GV, Thiessen-Philbrook HR, et al. Cardiovascular disease and hypertension risk in living kidney donors: an analysis of health administrative data in Ontario, Canada. Transplantation. 2008; Aug 1586(3):399-406.

[20] Mjoen G, Reisaeter A, Hallan S, et al. Overall and cardiovascular mortality in Norwegian kidney donors compared to the background population. Nephrol Dial Transplant. 2012;27(1):443-447.

[21] Mjoen G, Hallan S, Hartmann A, et al. Long-term risks for kidney donors. Kidney Int. 2014;86(1): 162167.

[22] Lewington S, Clarke R, Qizilbash N, et al. Age-specific relevance of usual blood pressure to vascular mortality: a meta-analysis of individual data for one million adults in 61 prospective studies. Lancet. 2002;360(9349):1903-1913.

[23] Boudville N, Prasad GV, Knoll G, et al. Meta-analysis: risk for hypertension in living kidney donors. Ann Intern Med. 2006;145(3):185-196.

[24] Kasiske BL, Anderson-Haag T, Israni AK, et al. A prospective controlled study of living kidney donors: three-year follow-up. Am J Kidney Dis. 2015;66(1): 114-124. 
[25] Levy D, Garrison RJ, Savage DD, et al. Prognostic implications of echocardiographically determined left ventricular mass in the Framingham Heart Study. N Engl J Med. 1990;322(22):1561-1566.

[26] Patel PC, Ayers CR, Murphy SA, et al. Association of cystatin $\mathrm{C}$ with left ventricular structure and function: the Dallas Heart Study. Circ Heart Fail. 2009;2(2):98-104.

[27] Levin A, Singer J, Thompson CR, et al. Prevalent left ventricular hypertrophy in the predialysis population: identifying opportunities for intervention. Am J Kidney Dis. 1996;27(3):347-354.

[28] Hewing B, Dehn AM, Staeck O, et al. Improved left ventricular structure and function after successful kidney transplantation. Kidney Blood Press Res. 2016;41(5):701-709.

[29] Moody WE, Ferro CJ, Edwards NC, et al. Cardiovascular effects of unilateral nephrectomy in living kidney donors. Hypertension. 2016;67(2): 368-377.

[30] Altmann U, Boger CA, Farkas S, et al. Effects of reduced kidney function because of living kidney donation on left ventricular mass. Hypertension. 2017;69(2):297303.

[31] Bellavia D, Cataliotti A, Clemenza F, et al. Long- term structural and functional myocardial adapta- tions in healthy living kidney donors: a pilot study. PloS one. 2015;10(11):e0142103.

[32] Shanahan CM, Crouthamel MH, Kapustin A, et al. Arterial calcification in chronic kidney disease: key roles for calcium and phosphate. Circ Res. 2011; 109(6):697-711.

[33] Herbelin A, Urena P, Nguyen AT, et al. Elevated circulating levels of interleukin-6 in patients with chronic renal failure. Kidney Int. 1991;39(5): 954960.

[34] Verbeke FH, Pannier B, Guerin AP, et al. Flowmediated vasodilation in end-stage renal disease. CJASN. 2011;6(8):2009-2015.

[35] Stam F, van Guldener C, Becker A, et al. Endothelial dysfunction contributes to renal functionassociated cardiovascular mortality in a population with mild renal insufficiency: the Hoorn study. JASN. 2006; 17(2):537-545.

[36] Barreto DV, Barreto FC, Liabeuf S, et al. Plasma interleukin-6 is independently associated with mor- tality in both hemodialysis and pre-dialysis patients with chronic kidney disease. Kidney Int. 2010;77(6): 550-556.

[37] Schmieder RE, Martus P, Klingbeil A. Reversal of left ventricular hypertrophy in essential hyperten- sion. A meta-analysis of randomized double-blind studies. JAMA. 1996;275(19):1507-1513.

[38] Mann JF, Gerstein HC, Pogue J, et al. Renal insufficiency as a predictor of cardiovascular outcomes and the impact of ramipril: the HOPE randomized trial. Ann Intern Med. 2001;134(8):629-636.

[39] Xie X, Liu Y, Perkovic V, et al. Renin-angiotensin system inhibitors and kidney and cardiovascular outcomes in patients with CKD: a Bayesian network meta-analysis of randomized clinical trials. Am J Kidney Dis. 2016;67(5):728-741.
[40] Kumar R, Singh VP, Baker KM. The intracellular renin-angiotensin system: implications in cardiovas- cular remodeling. Curr Opin Nephrol Hypertens. 2008;17(2):168-173.

[41] Kendi Celebi Z, Peker A, Kutlay S, et al. Effect of unilateral nephrectomy on urinary angiotensinogen levels in living kidney donors: 1 year follow-up study. J Renin Angiotensin Aldosterone Syst. 2017; 18(4):1470320317734082.

[42] Hathaway DK, Cashion AK, Milstead EJ, et al. Autonomic dysregulation in patients awaiting kidney transplantation. Am J Kidney Dis. 1998;32(2): 221-229.

[43] Ali A, Holm H, Molvin J, et al. Autonomic dysfunction is associated with cardiac remodelling in heart failure patients. ESC Heart Fail. 2018;5(1):46-52.

[44] Smith ER, Holt SG, Hewitson TD. alphaKlotho- FGF23 interactions and their role in kidney disease: a molecular insight. Cell Mol Life Sci. 2019.

[45] Gutierrez OM, Mannstadt M, Isakova $T$, et al. Fibroblast growth factor 23 and mortality among patients undergoing hemodialysis. N Engl J Med. 2008;359(6):584-592.

[46] Pavlovic D. The role of cardiotonic steroids in the pathogenesis of cardiomyopathy in chronic kidney disease. Nephron Clin Pract. 2014;128(1-2):11-21.

[47] Landstrom AP, Dobrev D, Wehrens X. Calcium signaling and cardiac arrhythmias. Circ Res. 2017; 120(12):1969-1993.

[48] Espe EK, Aronsen JM, Eriksen GS, et al. Assessment of regional myocardial work in rats. Circ Cardiovasc Imaging. 2015;8(2):e002695.

[49] Blacher J, Guerin AP, Pannier B, et al. Impact of aortic stiffness on survival in end-stage renal disease. Circulation. 1999;99(18):2434-2439.

[50] Westenberg JJ, van Poelgeest EP, Steendijk P, et al. Bramwell-Hill modeling for local aortic pulse wave velocity estimation: a validation study with velocityencoded cardiovascular magnetic resonance and invasive pressure assessment. J Cardiovasc Magn Reson. 2012;14(1):2.

[51] Schwartz T, Sanner H, Gjesdal O, et al. In juvenile dermatomyositis, cardiac systolic dysfunction is present after long-term follow-up and is predicted by sustained early skin activity. Ann Rheum Dis. 2014; 73(10):1805-1810.

[52] O'Leary DH, Polak JF, Kronmal RA, et al. Carotidartery intima and media thickness as a risk factor for myocardial infarction and stroke in older adults. N Engl J Med. 1999;340(1):14-22.

[53] Touboul PJ, Hennerici MG, Meairs S, et al. Mannheim carotid intima-media thickness and plaque consensus (2004-2006-2011). An update on behalf of the advisory board of the 3rd, 4th and 5th watching the risk symposia, at the 13th, 15th and 20th European Stroke Conferences, Mannheim, Germany, 2004, Brussels, Belgium, 2006, and Hamburg, Germany, 2011. Cerebrovasc Dis. 2012; 34(4):290-296.

[54] Hayano J, Sakakibara Y, Yamada A, et al. Accuracy of assessment of cardiac vagal tone by heart rate 
variability in normal subjects. Am J Cardiol. 1991; 67(2):199-204.

[55] Kleiger RE, Miller JP, Bigger JT, Jr, et al. Decreased heart rate variability and its association with increased mortality after acute myocardial infarction. Am J Cardiol. 1987;59(4):256-262.

[56] Giles D, Draper N, Neil W. Validity of the Polar V800 heart rate monitor to measure RR intervals at rest. Eur J Appl Physiol. 2016;116(3):563-571.

[57] Etehad Tavakol M, Fatemi A, Karbalaie A, et al. Nailfold capillaroscopy in rheumatic diseases: which parameters should be evaluated? BioMed Res Int. 2015;2015:1.

[58] Khan F, Patterson D, Belch JJ, et al. Relationship between peripheral and coronary function using laser Doppler imaging and transthoracic echocardiography. Clin Sci. 2008;115(9):295-300.

[59] Celermajer DS, Sorensen KE, Gooch VM, et al. Noninvasive detection of endothelial dysfunction in children and adults at risk of atherosclerosis. Lancet. 1992;340(8828):1111-1115.

[60] Thijssen DH, Black MA, Pyke KE, et al. Assessment of flow-mediated dilation in humans: a methodological and physiological guideline. Am J Physiol Heart Circ Physiol. 2011;300(1):H2-12.

[61] Laskey MA. Dual-energy X-ray absorptiometry and body composition. Nutrition. 1996;12(1):45-51.

[62] Hassanin N, Alkemary A. Early detection of subclinical uremic cardiomyopathy using two-dimensional speckle tracking echocardiography. Echocardiography. 2016;33(4):527-536.

[63] Puntmann VO, Voigt T, Chen Z, et al. Native T1 mapping in differentiation of normal myocardium from diffuse disease in hypertrophic and dilated cardiomyopathy. JACC Cardiovasc Imaging. 2013;6(4): 475-484.

[64] Nakamori S, Dohi K, Ishida $M$, et al. Native T1 mapping and extracellular volume mapping for the assessment of diffuse myocardial fibrosis in dilated cardiomyopathy. JACC Cardiovasc Imaging. 2018; 11(1):48-59.

[65] Ferreira VM, Piechnik SK, Dall'Armellina E, et al. Non-contrast T1-mapping detects acute myocardial edema with high diagnostic accuracy: a comparison to T2-weighted cardiovascular magnetic resonance. J Cardiovasc Magn Reson. 2012;14(1):42.

[66] Karamitsos TD, Piechnik SK, Banypersad SM, et al. Noncontrast T1 mapping for the diagnosis of cardiac amyloidosis. JACC Cardiovasc Imaging. 2013; 6(4):488-497.

[67] Arheden $H$, Saeed $M$, Higgins $C B$, et al. Measurement of the distribution volume of gadopentetate dimeglumine at echo-planar MR imaging to quantify myocardial infarction: comparison with 99mTc-DTPA autoradiography in rats. Radiology. 1999;211(3):698-708.

[68] Park SJ, Cho SW, Kim SM, et al. Assessment of myocardial fibrosis using multimodality imaging in severe aortic stenosis: comparison with histologic fibrosis. JACC Cardiovasc Imaging. 2019;12(1): 109119.

[69] Zou Z, Zhang HL, Roditi GH, et al. Nephrogenic systemic fibrosis: review of 370 biopsy-confirmed cases. JACC Cardiovascular imaging. 2011;4(11): 1206-1216.

[70] Gulani V, Calamante F, Shellock FG, et al. Gadolinium deposition in the brain: summary of evidence and recommendations. Lancet Neurol. 2017;16(7):564-570.

[71] Kellman P, Hansen MS. T1-mapping in the heart: accuracy and precision. J Cardiovasc Magn Reson. 2014;16(1):2.

[72] Espe EK, Aronsen JM, Skardal K, et al. Novel insight into the detailed myocardial motion and deformation of the rodent heart using high-resolution phase contrast cardiovascular magnetic resonance. JCardiovasc Magn Reson. 2013;15(1):82.

[73] Kuetting DL, Sprinkart AM, Dabir D, et al. Assessment of cardiac dyssynchrony by cardiac MR: a comparison of velocity encoding and feature track- ing analysis. J Magn Reson Imaging.. 2016;43(4): 940-946.

[74] Go AS, Chertow GM, Fan D, et al. Chronic kidney disease and the risks of death, cardiovascular events, and hospitalization. N Engl J Med. 2004;351(13): 1296-1305. 